\title{
Understanding Pathogen-host Interplay by Expression Profiles of LncRNA and mRNA in the Liver of Echinococcus Multilocularis-infected Mice
}

\section{Xiaofeng Nian}

LVRI: Lanzhou Veterinary Research Institute

\section{Li Li}

LVRI: Lanzhou Veterinary Research Institute

\section{Xusheng Ma}

LVRI: Lanzhou Veterinary Research Institute

\section{Xiurong Li}

LVRI: Lanzhou Veterinary Research Institute

Wenhui Li

LVRI: Lanzhou Veterinary Research Institute

Nianzhang Zhang

LVRI: Lanzhou Veterinary Research Institute John Asekhaen Ohiolei

LVRI: Lanzhou Veterinary Research Institute

\section{Le Li}

LVRI: Lanzhou Veterinary Research Institute

\section{Guodong Dai}

LVRI: Lanzhou Veterinary Research Institute Hongbin Yan

LVRI: Lanzhou Veterinary Research Institute

\section{Baoquan Fu}

LVRI: Lanzhou Veterinary Research Institute

\section{Sa Xiao}

Northwest A\&F University: Northwest Agriculture and Forestry University

Wanzhong Jia ( $\square$ jiawanzhong@caas.cn )

Lanzhou Veterinary Research Institute https://orcid.org/0000-0002-0801-7478

\section{Research}

Keywords: Echinococcus multilocularis, Alveolar echinococcosis, Long non-coding RNA, mRNA, Microarray, Innate immunity 
Posted Date: June 10th, 2021

DOl: https://doi.org/10.21203/rs.3.rs-589602/v1

License: (c) (i) This work is licensed under a Creative Commons Attribution 4.0 International License. Read Full License 


\section{Abstract}

Background: Echinococcus multilocularis $(E m)$ infection and the growth and proliferation of its metacestode within the internal organs of hosts are related to complex host-parasite interactions at the molecular level. Previous studies reported the profiles of long non-coding RNAs (IncRNAs) and mRNAs in Echinococcus granulosus-infected mice or cells, suggesting the potential role of IncRNAs in regulating host-parasite interplay. However, the profiles of IncRNAs and mRNAs of mice in response to Em are poorly understood.

Methods: Numerous differentially expressed IncRNAs (DELs) and mRNAs (DEMs) in the mouse liver at eight time points after Em infection were identified by microarray. Functional Annotation of dysregulated DEMs was conducted by gene ontology (GO) classification and the Kyoto Encyclopedia of Genes and Genomes (KEGG) enrichment analysis. The potential function of DELs was predicted by constructing IncRNA-mRNA co-expression network and Transcription factor (TF)-IncRNA-mRNA Ternary Network. Additionally, qRT-PCR and western blotting were used to validate the upregulated DEMs at 30 days postinfection (dpi), which were enriched in Toll-like and RIG--like receptor signaling pathways. Cytokines and chemokines involved in these two pathways were determined by ELISA.

Results: Thirty-one DEMs and 68 DELs were found continuously dysregulated. These DEMs were notably enriched in the "antigen processing and presentation," "Th1 and Th2 cell differentiation" and "Th17 cell differentiation" pathways. The potential function prediction of DELs revealed that most DELs might influence the differentiation of Th17 cell and TGF- $\beta /$ Smad pathway through trans regulating the SMAD3, STAT1, and early growth response (EGR) genes. Additionally, the validated results by qRT-PCR and western blotting showed that the mRNA expression levels of these genes increased while the corresponding protein expression levels were unaltered except c-Jun amino-terminal kinase (JNK). Regardless, phospho-nuclear factor Kappa B (p-NF-кB) downstream of these two pathways was induced at 15 and $30 \mathrm{dpi}$, which led to the elevated levels of IL-1 beta and IL-6 in the serum.

Conclusion: Our data provide novel clues in understanding the roles of IncRNAs in the host-Em interplay and the influence of Em infection on host innate immunity.

\section{Background}

Echinococcus multilocularis (Em), one of the small tapeworms of foxes whose metacestodes reside mostly in the liver of rodents (common intermediate host) or occasionally human beings, causes alveolar echinococcosis $(\mathrm{AE})$ that is characterized by a long-term, tumor-like manifestation [1]. $A E$ is a severe zoonosis prioritized alongside other neglected tropical diseases by the World Health Organization [2]. Mortality can reach more than $90 \%$ in untreated or poorly treated AE patients after diagnosis within 10 to 15 years [3], resulting in the loss of about 666,434 disability-adjusted life years (DALYs) annually [4]. During the course of this infection, the growth and proliferation of Em metacestode within the internal 
organs of hosts are related to complex host-parasite interactions, and the interplay between parasite and host at the molecular level remains to be fully understood [5].

Long non-coding RNAs (IncRNAs) have received increased attention lately as a newly discovered class of regulatory molecules [6-8]. Studies have shown that the expression profile of IncRNA in the host can be altered during viral, bacterial, or parasitic infection, suggesting the potential role of IncRNAs in regulating host-pathogen interactions [9-12]. Moreover, a growing body of evidence showed that some dysregulated IncRNAs of the host could participate in the pathophysiological process of various diseases by affecting the host immune mechanisms [13-15]. For instance, host Lnczc3h7a can bind to TRIM25, playing the role of a molecular scaffold to stabilize the RIG-I-TRIM25 complex and promote the host's innate immune responses [16]. A decreased level of host IncRNA AK189470.1 after viral infection can promote viral replication by reducing glutathione S-transferase M1 (GSTM1)-mediated S-

glutathionylation of TBK1 [17]. A study of parasite infection showed that Cryptosporidium could upregulate the host IncRNA NR_033736, suppressing type I IFN-mediated gene transcription, and promoting the epithelial innate defense against the infection $[18,19]$.

Until now, the host IncRNA profiles after Echinococcus infection were mainly identified in Echinococcus granulosus-infected mice or cells [20-22]. However, the host IncRNA expression pattern and functional activities in response to Em infection are poorly investigated. Here, we explored the expression profiles of IncRNAs and mRNAs in Em-infected mouse liver at eight points [2, 4, 8, 15, 30, 60, 90 and 150 days postinfection (dpi)] by microarray. It was found that the continuously dysregulated IncRNAs across all time points had a possible regulatory role in the TGF- $\beta$ /Smad pathway and Treg/Th17 imbalance through trans regulating some transcription factors (TF) in AE. Additionally, our validation results of some differentially expressed mRNAs (DEMs) improved our understanding of host innate immunity in response to $E m$ infection. These IncRNAs and genes could be good potential targets for further investigation.

\section{Methods}

\section{Mice and experimental design}

The metacestodes were obtained from BALB/c mice intraperitoneally infected with protoscolices from a naturally infected plateau pika (Ochotona curzoniae) in Yushu, Qinghai province, China (See Additional document for methods).

A total of 48 pathogen-free female BALB/c mice weighing around $25 \mathrm{~g}$ were purchased from Lanzhou University, Lanzhou, China, and divided randomly into two groups (24 mice each). One group was used as the case (CA), while the other served as the control (Con). They were housed in cages with a $12 \mathrm{~h}$ light/dark cycle and provided with food and water adlibitum during the entire experimental period. After being normally fed for two weeks, all mice in the CA group were injected intraperitoneally with $100 \mu \mathrm{l}$ of protoscolex suspension containing about 1,000 Em protoscolices, while all mice in the Con group were given the same volume of sterile saline solution using the same surgical procedure. Each group was 
randomly divided into eight sub-groups (three mice per subgroup in separate cages) corresponding to eight autopsy time points $(2,4,8,15,30,60,90$ and $150 \mathrm{dpi})$.

\section{Sample collection}

At 2, 4, 8, 15, 30, 60, 90 and $150 \mathrm{dpi}$, tail venous blood samples and liver samples were collected from the three animals in CA and Con groups. The blood samples were allowed to clot at room temperature for 30 min, followed by centrifugation at $3,500 \mathrm{rpm}$ for $15 \mathrm{~min}$ at $20^{\circ} \mathrm{C}$. The serum layer was collected, divided into aliquots and frozen at $-80^{\circ} \mathrm{C}$ until use. In CA mice, samples of the liver were carefully taken from the periparasitic liver tissue (relatively close to the lesion to avoid contamination by Em tissue/cells) and immediately frozen in liquid nitrogen. Control samples were taken from the corresponding liver lobe of Con mice.

\section{RNA preparation and microarray}

The IncRNA and mRNA expression patterns of three CA mice and three Con mice at 2, 4, 8, 15, 30, 60, 90 and $150 \mathrm{dpi}$ were detected. The Agilent Mouse IncRNA Microarray V3 (4*180 K, Design ID: 084388) was used in this experiment and data analysis of the 48 samples was conducted by OE Biotechnology Co., Ltd. (Shanghai, China). Total RNA was obtained using a commercially available Kit (mirVana miRNA Isolation Kit, Ambion, AM1561) following the manufacturer's instruction and quantified by NanoDrop ND2000 (Thermo Scientific, USA), while the Agilent Bioanalyzer 2100 (Agilent Technologies, USA) was used to examine RNA integrity. The sample labeling, microarray hybridization and washing were done based on standard protocols according to the manufacturer. Briefly, total RNA was transcribed to double-strand cDNAs, followed by synthesis into cRNAs, and then labeled with Cyanine-3-CTP. The labeled cRNAs were hybridized onto the microarray. After washing, the scanning of the arrays was achieved by Agilent Scanner G2505C (Agilent Technologies, USA).

\section{Data pre-processing}

Analysis of the array images to get the raw data was conducted by Agilent Feature Extraction software (version10.7.1.1, Agilent Technologies, USA). Genespring (version 14.8, Agilent Technologies, USA) was employed to finish the basic analysis with the raw data. First, the raw data were normalized with the quantile algorithm. Probes with at least one of two conditions flagged in " $P$ " were chosen for further data analysis. The $t$-test was used and differentially expressed genes or IncRNAs were identified through Fold change (FC) and $p$-value filtering.

\section{DELs and DEMs analyses}

LncRNA and mRNA expression levels between the CA and Con groups at each autopsy time point $(2,4,8$, $15,30,60,90$ and $150 \mathrm{dpi}$ ) were observed. Significant differences were determined by $t$-test to identify DELs and DEMs. Then, the IncRNAs and mRNAs with values of $p<0.05$ as well as $|F C| \geq 2$, were filtrated as DELs and DEMs. The expression pattern of DELs and DEMs among groups was shown by a Hierarchical Clustering display. 


\section{Functional annotation}

To provide insight into the biological functions of the differentially expressed genes, gene ontology (GO) classification (http://geneontology.org/) and the Kyoto Encyclopedia of Genes and Genomes (KEGG) enrichment analysis (https://www.kegg.jp/) were conducted. All three categories of GO term (biological process, cellular components, and molecular function) were analyzed, with a $p$-value $<0.05$ as the threshold for selecting significantly enriched functional GO terms and KEGG pathways.

\section{Construction of IncRNA-mRNA co-expression network}

Pearson correlation coefficient (PCC) was used to describe the co-expression relationship between IncRNAs and mRNAs. For each IncRNA, we calculated the PCC value of its expression level with the expression level of each mRNA. LncRNA-mRNA pairs with $\mid \mathrm{PCC}$ value $\geq 0.7$ and $p<0.05$ were filtrated to construct the network, which was displayed by Cytoscape 3.7.1 (http://cytoscape.org/).

\section{Cis and trans regulating target prediction of IncRNAs and construction of TF-IncRNA-mRNA ternary network}

For each IncRNA, we identified the mRNAs as "cis-regulated mRNAs" when: (1) the mRNAs loci are within a $100 \mathrm{~kb}$ window up- or downstream of the given IncRNA, (2) the PCC of IncRNA-mRNA expression is significant ( $p$-value of correlation $\leq 0.05$ ) [23]. To further understand the role of IncRNAs in AE, the TF related to IncRNAs based on the cumulative hypergeometric test was used to construct a co-expression network with DELs. Each IncRNA could correlate with one or more TFs and each pair of IncRNA-TF results from several gene enrichment. Then, we introduced the mRNAs that were co-expressed with these IncRNAs and selected the top 10 by $p$-value ranking to construct the network of TF-IncRNA-mRNA [2426].

\section{Validation by quantitative real-time PCR (qRT-PCR)}

Each liver sample was processed and analyzed separately. Total RNA was extracted from liver samples (about $50 \mathrm{~mm}^{3}$ ) of CA and Con mice using mirVana miRNA Isolation Kit (Ambion, AM1561). Total RNA (1 mg) was transcribed to cDNA followed by qRT-PCR using PrimeScript ${ }^{\mathrm{TM}} \mathrm{RT}$ reagent Kit with gDNA Eraser (Takara Bio Inc., RR047A) in a CFX96 instrument (Bio-Rad Laboratories, Inc.). The reaction conditions for the qRT-PCR included an initial denaturation step at $95^{\circ} \mathrm{C}$ for $30 \mathrm{~s}$, and 40 cycles of $10 \mathrm{~s}$ at $95^{\circ} \mathrm{C}$ and 1 min at $60^{\circ} \mathrm{C}$. All experiments were operated in triplicate and $\beta$-actin was used for normalization, using the $2^{-\triangle \triangle C T}$ method to calculate the relative expression levels. The PCR primers used in this study can be found in the Additional Table for methods.

\section{Protein extraction and western blotting (WB)}

The total protein from the liver was extracted using a Tissues Total Protein Extraction Kit for WB (invent, USA). Briefly, the proteins were transferred onto PVDF membranes (Bio-Rad, Hercules, CA, USA) and blocked with 5\% skim milk in TBST ( $25 \mathrm{mM}$ Tris-HCl, $125 \mathrm{mM} \mathrm{NaCl}, 0.1 \%$ Tween 20) for $1 \mathrm{~h}$ at $37^{\circ} \mathrm{C}$. Then, 
IRAK4, IKKB, MKK, JNK, NLRX1, and p-NF-KB primary antibodies (Abcam, Cambridge, MA, USA) were incubated at $4^{\circ} \mathrm{C}$ overnight. After washing 5 times with TBST, the membranes were incubated with peroxidase-conjugated secondary antibody (Zhongshan Golden Bridge, Beijing, China) for $1 \mathrm{~h}$. $\beta$-actin was used as an internal control.

\section{Measurement of cytokines and chemokines}

The levels of tumor necrosis factor- $a$ (TNF- $\alpha$ ), interferon alpha (IFN- $\alpha$ ), interferon beta (IFN- $\beta$ ), interleukin-1 beta (IL-1 $\beta$ ), interleukin-6 (IL-6), interleukin-12 (IL-12), CXCL10, CCL5, and CCL3 at 2, 4, 8, 15, and 30 dpi in the serum were determined by the LEGENDplex Mouse Anti-Virus Response (13-plex) Panel (740621, Biolegend) and ELISA according to the manufacturer's instructions. The data were read on an LSRFortessa (BD Biosciences). CCL3 was determined by ELISA (Mouse CCL3/MIP1 alpha AccuSignal ELISA Kit-KOA0258, Rockland, USA) following the manufacturer's instructions. The OD of $450 \mathrm{~nm}$ (OD450) minus the background of plate absorbance was read on an ELISA microplate reader (BIO-RAD, Model 680).

\section{Statistical analysis}

PCC was used to calculate the correlation between IncRNAs and mRNAs (IPCC valuel $\geq 0.7$ ). The Student's $t$-test was applied to calculate the statistical difference of qRT-PCR results and concentration of cytokines and chemokines in serum using GraphPad Prism 8. All results were considered statistically significant when $p$-values were $<0.05$.

\section{Results}

\section{Confirmation of Em infection in BALB/c mice and identification of DEMs and DELs}

Em infection was confirmed in all challenged mice by observing gross pathological lesions. The infected livers were enlarged, and the surface of the liver lobes was characterized by translucent or whitish alveolar vesicles.

To explore the mRNA and IncRNA expression profiles of mouse liver in response to Em during the whole infection period, we selected eight time points to estimate the transcript expression levels between CA and Con groups by microarray analyses. At each time point, CA and Con groups were both tested using three biological replicates. The workflow for the transcript analysis is shown in Fig. 1a. The mRNAs and IncRNAs with an expression change of more than two-fold $(F C \geq 2)$ and $p<0.05$ were selected as differentially expressed candidates. As shown in Table 1, the numbers of DEMs and DELs were detected for the corresponding Con at the eight time points. Notably, for DEMs, the proportion of downregulated genes was from $51.3 \%$ to $68.2 \%$ at seven time points except for $30 \mathrm{dpi}$ (downregulated genes were $25.1 \%$ ). For DELs, there were more downregulated genes than upregulated genes at all the eight time points, with a proportion of $58.4 \%$ to $83.7 \%$. In general, the changes of DELs and DEMs displayed a similar trend. Furthermore, the volcano plots (See Additional Fig. S1a, b) and the hierarchical clustering 
plots (See Additional Fig. S1c, d) showed that the expression patterns of mRNAs and IncRNAs between the $\mathrm{CA}$ and Con groups at the eight time points were significantly different. These results suggested that $E m$ is a strong trigger that can alter the expression of numerous mRNAs and IncRNAs at different stages during the infection course. More DEMs and DELs identified at 4, 15, 60, 90, 150 dpi indicates that these time points are representative of transcriptome change times.

To investigate the biotype of identified DELs, according to the position of IncRNA in the genome relative to protein-encoding gene, DELs at each autopsy time point were divided into four categories (http://www.noncode.org/). As shown in Fig. 1b, more than 78\% IncRNA were lincRNAs, antisense IncRNA and sense no exonic IncRNA accounted for the nearly equal proportion (7-9\%), while exonic IncRNAs were fewest $(\sim 3 \%)$. The data demonstrated that biotypes of the DELs and their corresponding ratios at different time points during the Em infection course were almost the same.

\section{Validation of candidate DEMs and DELs using qRT-PCR}

To validate the DELs/DEMs identified in mouse livers at each autopsy time point by microarray, twenty DEMs and DELs (10 each) randomly selected were verified by qRT-PCR. As shown in Fig. 2 , the qRT-PCR results of 10 mRNAs (six upregulated and four downregulated) and 10 IncRNAs (six upregulated and four downregulated) confirmed the microarray data, showing similar trends in the up- or downregulated mRNAs (Fig. 2a) and IncRNAs (Fig. 2b).

\section{Functional annotation of thedifferentially expressed mRNAs and overall gene expression changes in Em- infected mouse liver}

To better understand the biological roles of differentially expressed mRNAs at each autopsy time point, the up- and downregulated DEMs were estimated by KEGG pathway analysis. In Fig. 3a, it was found that two innate immunity pathways, Toll-like (TLRs) and RIG--llike receptor (RLRs) signaling pathway, were included in the significantly enriched KEGG terms (top 30) of the DEMs at $30 \mathrm{dpi}$. The results showed that IRAK4, IKK $\beta$, MKK, JNK, and IFN-a were upregulated in TLRs signaling pathway (Additional Fig. S2a), and NLRX1, IKK $\beta$, JNK, and IFN-a were upregulated in RLRs signaling pathway (Additional Fig. S2b).

Additionally, the KEGG analysis of downregulated DEMs showed that the ubiquitin-mediated proteolysis pathway was included in the significantly enriched KEGG terms at seven time points $(2,4,8,15,30,60$, $150 \mathrm{dpi}$ ). Notably, there were more dysregulated genes in this pathway at $15 \mathrm{dpi}$ (Additional Fig. S2c). This result indicated that $E m$ infection had a broad and long-lasting impact on this pathway of the host.

Additionally, to search the genes with continued aberrant expression during the infection course, 68 DELs (downregulated) and 31 DEMs (21 downregulated and 10 upregulated) were found overlapped at all time points by Venn analysis (Additional Table S1a, b). Then, GO analysis was performed to understand the potential functions of these shared DEMs during the whole Em infection period. In Fig. 3b, the results showed that these DEMs were significantly enriched in GO terms related to BP, such as "antigen processing and presentation of peptide, polysaccharide or exogenous peptide antigen via MHC class II," "response to interferon-gamma" and "negative regulation of T cell proliferation"; most shared DEMs were 
associated with GO terms related to CC, including "MHC class II protein complex," "brush border" and "Iysosomal membrane"; these shared DEMs were enriched in MF associated with transportation, including "peptide antigen binding," "symporter activity" and "transporter activity."

Next, we conducted 31 shared DEMs by the KEGG database to determine the signal transduction and/or metabolic pathways of these dysregulated mRNAs to understand the host-parasite interplay further. As shown in Fig. 3c, these overlapped DEMs throughout the infection course were mainly involved in "antigen processing and presentation," "intestinal immune network for IgA production," "Th1 and Th2 cell differentiation," and "Th17 cell differentiation," suggesting that Em infection had an enduring influence on these signaling pathways. They were continuously active throughout the infectious course.

\section{Construction of co-expression network between overlapped DELs and DEMs}

To demonstrate the interactive relationship between the overlapped DELs and DEMs, 31 shared DEMs and 68 shared DELs containing 2,108 relationships were estimated by calculating PCC values. According to the cutoff criteria $(p<0.05$ and $|\mathrm{PCC}|>0.7)$, Fig. 4 showed the IncRNA-mRNA co-expression network of the top five IncRNAs that had the most co-expression relationships with mRNAs. From the network, it was observed that NONMMUT099399.1 had the most relationships with DEM, showing that more than one mRNA was predicted to be regulated by one IncRNA. In turn, one mRNA could correspond to several IncRNAs, for example, mRNA Cers6 may be regulated by three IncRNAs (NONMMUT037651.2, NONMMUT099399.1, and NONMMUT125804.1). Furthermore, these five IncRNAs were co-expressed with 26 DEMs, which accounted for $84 \%$ of the total shared DEMs, indicating that these IncRNAs are the candidates with the highest potential for being involved in the signaling pathways enriched by the 31 shared DEMs as displayed by KEGG analysis.

\section{Cis and trans regulating target prediction of overlapped IncRNAs and construction of IncRNA-TF-mRNA network}

Generally, IncRNAs perform functions by interacting with their targets, so we predicted the cis- and transtarget gene potential of these IncRNAs. We searched for protein-coding genes (among the 31 shared DEMs) $10-\mathrm{kb}$ up- and downstream of the 68 shared DELs and found no IncRNAs that were transcribed near $(<10 \mathrm{~kb})$ the 31 DEMs neighbors. Then, the trans regulatory functions of IncRNAs were predicted by evaluating the relationship between the TFs and IncRNAs based on the cumulative hypergeometric test. We found that among the 68 shared IncRNAs, 41 IncRNAs corresponded to 16 TFs (EGR1, EGR2, EGR3, EGR4, STAT1, STAT5A, SMAD3, MYC, HINFP, ONECUT2, P2RX5, NR5A2, TRP53, MAX, PITX2, HIC1), which could regulate the expression of IncRNAs. In Fig. 5a, among the 16 TFs, EGR2 had the most trans relationships with DELs. EGR1, EGR3, and EGR4 were also related to the shared DELs, indicating that the EGR family may play an important role on the host DELs during Em infection. Then, since each IncRNATF pair results from several gene enrichment, we selected the top 10 co-expressed mRNAs by $p$-value ranking to further build a IncRNA-TF-mRNA ternary network (Fig. 5b). 


\section{Validation of six up-regulated DEMs involved in Toll-like and RIG-H-like receptor signaling pathways by qRT-PCR and WB}

To further investigate the influence of Em infection on host innate immunity, six upregulated DEMs (IRAK4, IKKß, MKK, JNK, IFN-a, and NLRX1) enriched in TLRs and RLRs signaling pathway in KEGG analysis were validated by qRT-PCR. In Fig. $6 a$, the results showed that expression levels of all these genes increased in the liver of CA mice compared to the control at 15 and $30 \mathrm{dpi}$, consistent with the microarray data (Additional Fig. S3). Then, the protein expression of IKK 3 , JNK, MKK, IRAK4, and NLRX1 was determined by WB. Compared with the Con groups, the protein expression of IKK $\beta$, MKK, IRAK4, and NLRX1 were unaltered at 15 and $30 \mathrm{dpi}$ except for c-Jun amino-terminal kinase (JNK), which decreased at $30 \mathrm{dpi}$. And importantly, the protein expression of phospho-nuclear factor Kappa B (p-NF-KB) increased compared to the control at 15 and $30 \mathrm{dpi}$ (Fig. 6b). Taken together, these results demonstrated that $E m$ infection induced mRNA expression of some key components in TLRs and LRLs signaling pathway, but their protein expression levels were not altered, suggesting that the changes in the expression level of these genes during transcription and translation were inconsistent and may be due to the parasite effect on post-transcriptional regulation. In any case, downstream these two innate immune pathways, p-NF-kB, a master regulator of key inflammatory gene expression, was induced at 15 and $30 \mathrm{dpi}$.

\section{Determination of cytokines and chemokines downstream TLRs and RLRs signaling pathway}

To assess the impact of Em infection on the cytokines and chemokines downstream of TLRs and RLRs signaling pathway shown by KEGG database analyses (See Additional Fig. S2a, b), the serum levels of IL1 $\beta$, IL-6, CCL5 (RANTES), TNF-a, IL-12, CCL3 (MIP1-a), IFN-a, IFN- $\beta$, CXCL10 (IP10) were determined at 2, 8, 15 and $30 \mathrm{dpi}$. The results showed that IL-1 $\beta$, IL-6, TNF-a, and CXCL10 increased significantly at $30 \mathrm{dpi}(p$ $<0.05)$. IFN- $\alpha$, IFN- $\beta$, IL-12, CCL5, and CCL3 demonstrated an increasing trend but the differences were not statistically significant compared to the control (Fig. 7). Although not all the factors were significantly elevated, there was a clear underlying trend. Furthermore, among these, as the most important proinflammatory cytokines, levels of IL-1 $\beta$ and IL- 6 in serum increased markedly at $30 \mathrm{dpi}$, which may be due toincreased levels of p-NF-KB at 15 and $30 \mathrm{dpi}$ as evident in the WB results.

\section{Discussion}

The potentially lethal zoonotic disease AE is caused by the metacestode larval stages of the tapeworm $E m$. During the growth and proliferation of metacestode within the host liver, molecular interactions of host-parasite take place [27]. Therefore, elucidating the complex interplay between the different biological macromolecules of the host and parasite is critical to understand host-pathogen relationships better.

LncRNAs are the most prevalent and functionally diverse class of RNAs, interacting with protein, DNA and RNA to play their roles in epigenetics, transcription and post-transcriptional regulation [28]. They are suggested to be associated with both cause and progression of various diseases [29]. The function and 
range of IncRNA-mediated regulation in hosts following pathogen infection are increasingly appreciated recently [30].

Considering the pathogenesis of chronically developing $A E$, eight time points $(2,4,8,15,30,60,90$ and $150 \mathrm{dpi}$ ) along the infection course were selected to survey host IncRNAs and mRNAs profiles to capture a preliminarily and comprehensively overview of this important class of regulatory molecules in AE. Sixtyeight IncRNAs were found continuously dysregulated during the infection course. Among them, top five IncRNAs (NONMMUT103521.1, NONMMUT099399.1, NONMMUT105538.1, NONMMUT125804.1, NONMMUT037651.2) had co-expression relationships with $84 \%$ of the shared DEMs (total 31 shared DEMs) that were mainly responsive to "Th17 cell differentiation," "Th1 and Th2 cell differentiation," and "antigen processing and presentation," among others, indicating that these IncRNAs may take part in these host pathways in the infection process. Furthermore, the trans regulating targets prediction analysis of these overlapped DELs revealed that a few TFs might be the interacting targets of DELs, including EGR genes, Trp53 (p53), Smad3, MAX, STAT1 and STAT5A, which may be also be involved in these pathways. More than 20 IncRNAs had trans relationships with EGR2 that influenced Th17 cytokine expression and the differentiation of Th17 cells [31]. Studies showed that Th17 cells played a significant role during the Em infection period and were related to immunopathology in $A E[32,33]$. Th17 differentiation was related to the imbalance of Treg/Th17, which is observed at mid and late-stage after Em infection and related to Th1/Th2-cell balance during Em infection [34]. Additionally, studies have shown that the Treg/Th17 imbalance that appeared during the $E m$ infection was regulated by the TGF- $\beta / S m a d$ pathway [35]. The TGF- $\beta /$ Smad pathway can drive the expansion of Foxp3 ${ }^{+}$Treg in the host after Em infection [36-38]. Therefore, it appears that these DELs may play a regulatory role though EGR2 and Smad3, and thus be involved in Th17 cell differentiation and TGF- $\beta /$ Smad pathway in AE. Based on the above observations, we hypothesized a schematic presentation to show the possible regulatory targets of these candidate DELs during Em infection (Fig. 8a). The functional mechanism of these candidate IncRNAs with Smad3 and/or EGR2 in regulating the TGF- $\beta$ /Smad pathway and the influences on Treg and Th17 subsets warrants further investigation.

The KEGG analysis results of DEMs agreed with the previous findings. At the early stage ( $30 \mathrm{dpi})$, the dysregulated mRNAs were mainly involved in processes such as "immune response," "inflammatory response," and "antigen processing and presentation." At the middle stage (90 dpi), the upregulated DEMs were enriched in "transport," "MAPK signaling pathway," and "cell adhesion." During the late stage (150 dpi), the DEMs were related to metabolic pathways, such as "nitric oxide biosynthetic process," "lipid metabolism," and "central carbon metabolism" [39, 40]. Elevated levels of IL- $1 \beta$, IL- 6 in the sera at 30 dpi confirmed the induction of the inflammatory response, which requires the activation of pattern recognition receptor (PRR). Here, TLRs and RLRs signaling pathways enriched by the upregulated DEMs at $30 \mathrm{dpi}$ had attracted our attention. Previous studies showed significantly elevated TLR2 and TLR4 mRNA in AE patients where they help the parasite evade host immunity. The TLR2 may also play a role in regulating tissue infiltrative growth in $A E$ and facilitating the long-term survival of the parasite in the host $[41,42]$. We measured the mRNA and protein expression levels of upregulated DEMs (IRAK4, IKK $\beta$, MKK, 
JNK, and NLRX1) in microarray results, which were enriched in TLRs and RLRs signaling pathways, to validate the effect of $E m$ on these two innate immune pathways further. These increased mRNA expression levels of these genes confirmed that $E m$ had influenced the key components in TLRs and RLRs signaling pathways of the host. However, their protein expression levels were unaltered. The discrepancies between mRNA expression and protein expression regarding these genes may be explained by post-transcriptional events, and deserve further studies. Such events could result from parasite components/secretions, favoring an attenuated innate immune response and contributing to the parasite growth. Also, it has been reported that RNA molecules can be transferred to recipient cells by extracellular vesicles (EVs) [43,44], and EVs released by Em metacestodes can regulate the expression of cytokines and key components in the LPS/TLR4 pathway in vitro [45]. So it is possible that these RNAs were recognized by RIG-I receptor to activate the RLRs pathway, further modulating the innate immunity of the host $[46,47]$. Accordingly, we hypothesized in a schematic presentation the host-Em relationship and the involvement of the TLRs and RLRs signaling pathway (Fig. 8b).

\section{Conclusion}

Overall, our results provide a molecular biology foundation to further comprehend the roles of IncRNAs in the host-Em interplay, and offer novel clues in understanding the influence of Em infection on host innate immunity and the interaction mechanisms between $E m$ and the host, which will help in the future to design new antiparasitic strategies targeting the host's non-protein-coding genome.

\section{Abbreviations}

AE Alveolar echinococcosis

Em Echinococcus multilocularis

DALYs Disability-adjusted life years

LncRNAs Long non-coding RNAs

DELs Differentially expressed IncRNAs

DEMs Differentially expressed mRNAs

EGR Early growth response

dpi Days post-infection

PCC Pearson correlation coefficient

BP Biological process

CC Cellular component 
MF Molecular function

TLR Toll-like receptor

RLR RIG-I-like receptor

TF Transcription factor

JNK C-Jun amino-terminal kinase

\section{Declarations}

Ethics approval Animal experiments were conducted in compliance with the recommendations in the Guide for the Care and Use of Laboratory Animals and were approved by Lanzhou Veterinary Research Institute, Chinese Academy of Agricultural Sciences Ethics Committee. Animal handling was strictly carried out according to the animal protection laws of the People's Republic of China (A Draft, Sep. 18, 2009).

Consent for publication Not applicable.

Availability of data and material The datasets used and/or analyzed during the current study are available from the corresponding author on reasonable request.

Competing interests The authors declare no competing interests.

Funding This work was funded by the National Key Research and Development Plan (2017YFD0501301; 2018YFC1602504), Central Public-Interest Scientific Institution Basal Research Fund (1610312020016), and Cultivation of Achievements of State Key Laboratory of Veterinary Etiological Biology (SKLVEB2020CGPY01).

Authors' contributions XFN and HBY designed the experiments and drafted the manuscript. XFN, LL, XSM, $X R L, G D D$ and LL carried out animal experiments, qRT-PCR and WB. XFN, WHL and NZZ analyzed experimental results and data. WZJ and SX guided the design of the study and supervised XFN. JAO and WZJ revised the manuscript. WZJ supervised the overall study. All authors read and approved the final manuscript.

Acknowledgements We thank Shanghai OE biotech Co., Ltd., China for the gene microarray analysis, and the support of bioinformatics analysis.

\section{References}

1. Garcia HH, Moro PL, Schantz PM. Zoonotic helminth infections of humans: echinococcosis, cysticercosis and fascioliasis. Curr Opin Infect Dis. 2007;20:489-94. 
2. World Health Organization. (2020) Echinococcosis. https://www.who.int/news-room/factsheets/detail/echinococcosis. Accessed 15 July 2020.

3. McManus DP, Gray DJ, Zhang WB, et al. Diagnosis, treatment, and management of echinococcosis. BMJ. 2012;344:e3866.

4. Torgerson PR, Keller K, Magnotta M, et al. The global burden of alveolar echinococcosis. PLoS Negl Trop Dis. 2010;4:e722.

5. Budke CM, Casulli A, Kern P, et al. Cystic and alveolar echinococcosis: Successes and continuing challenges. PLoS Negl Trop Dis. 2017;11:e0005477.

6. Batista PJ, Chang HY. Long noncoding RNAs: cellular address codes in development and disease. Cell. 2013;152:1298-307.

7. Wang KC, Yang YW, Liu B, et al. A long noncoding RNA maintains active chromatin to coordinate homeotic gene expression. Nature. 2011;472:120-4.

8. Engreitz JM, Ollikainen N, Guttman M. Long non-coding RNAs: spatial amplifiers that control nuclear structure and gene expression. Nat Rev Mol Cell Biol. 2016;17:756-70.

9. Liu WW, Ding C. Roles of LncRNAs in Viral Infections. Front Cell Infect Microbiol. 2017;7:205.

10. Menard KL, Haskins BE, Denkers EY. Impact of Toxoplasma gondii Infection on Host Non-coding RNA Responses. Front Cell Infect Microbiol. 2019;9:132.

11. Ahmed W, Liu ZF. Long Non-Coding RNAs: Novel Players in Regulation of Immune Response Upon Herpesvirus Infection. Front Immunol. 2018;9:761.

12. Saco A, Rey-Campos M, Novoa B, et al. Transcriptomic Response of Mussel Gills After a Vibrio splendidus Infection Demonstrates Their Role in the Immune Response. Front Immunol. 2020;11:615580.

13. Gomes CPC, Spencer H, Ford KL, et al. The Function and Therapeutic Potential of Long Non-coding RNAs in Cardiovascular Development and Disease. Mol Ther Nucleic Acids. 2017;8:494-507.

14. Yang B, Xia ZA, Zhong BW, et al. Distinct Hippocampal Expression Profiles of Long Non-coding RNAs in an Alzheimer's Disease Model. Mol Neurobiol. 2017;54:4833-46.

15. Yarani R, Mirza AH, Kaur S, et al. The emerging role of IncRNAs in inflammatory bowel disease. Exp Mol Med. 2018;50:1-14.

16. Lin HY, Jiang MH, Liu L, et al. The long noncoding RNA Lnczc3h7a promotes a TRIM25-mediated RIG-I antiviral innate immune response. Nat Immunol. 2019;20:812-23.

17. Wang YJ, Wang P, Zhang YK, et al. Decreased Expression of the Host Long-Noncoding RNA-GM Facilitates Viral Escape by Inhibiting the Kinase activity TBK1 via S-glutathionylation. Immunity. 2020;53:1168-81.e7.

18. Li M, Gong AY, Zhang XT, et al. Induction of a Long Noncoding RNA Transcript, NR_045064, Promotes Defense Gene Transcription and Facilitates Intestinal Epithelial Cell Responses against Cryptosporidium Infection. J Immunol. 2018;201:3630-40. 
19. Li J, Jin KH, Li M, et al. A host cell long noncoding RNA NR_033736 regulates type I interferonmediated gene transcription and modulates intestinal epithelial anti-Cryptosporidium defense. PLoS Pathog. 2021;17:e1009241.

20. Yu AP, Wang Y, Yin JH, et al. Microarray analysis of long non-coding RNA expression profiles in monocytic myeloid-derived suppressor cells in Echinococcus granulosus-infected mice. Parasit Vectors. 2018;11:327.

21. Zhang XF, Gong WC, Cao SK, et al. Comprehensive Analysis of Non-coding RNA Profiles of ExosomeLike Vesicles From the Protoscoleces and Hydatid Cyst Fluid of Echinococcus granulosus. Front Cell Infect Microbiol. 2020;10:316.

22. Lu Y, Liu H, Yang XY, et al. Microarray Analysis of IncRNA and mRNA Reveals Enhanced Lipolysis Along With Metabolic Remodeling in Mice Infected With Larval Echinococcus granulosus. Front Physiol. 2020;11:1078.

23. Kopp F, Mendell JT. Functional Classification and Experimental Dissection of Long Noncoding RNAs. Cell. 2018;172:393-407.

24. Ernst J, Plasterer HL, Simon I, et al. Integrating multiple evidence sources to predict transcription factor binding in the human genome. Genome Res. 2010;20:526-36.

25. Guttman M, Rinn JL. Modular regulatory principles of large non-coding RNAs. Nature. 2012;482:33946.

26. Gerstein MB, Kundaje A, Hariharan M, et al. Architecture of the human regulatory network derived from ENCODE data. Nature. 2012;489:91-100.

27. Brehm K, Koziol U. Echinococcus-Host Interactions at Cellular and Molecular Levels. Adv Parasitol. 2017;95:147-212.

28. Ma LN, Bajic VB, Zhang Z. On the classification of long non-coding RNAs. RNA Biol. 2013;10:92533.

29. Mishra S, Verma SS, Rai V, et al. Long non-coding RNAs are emerging targets of phytochemicals for cancer and other chronic diseases. Cell Mol Life Sci. 2019;76:1947-66.

30. Shirahama S, Miki A, Kaburaki T, et al. Long Non-coding RNAs Involved in Pathogenic Infection. Front Genet. 2020;11:454.

31. Miao T, Raymond M, Bhullar P, et al. Early growth response gene-2 controls IL-17 expression and Th17 differentiation by negatively regulating Batf. J Immunol. 2013;190:58-65.

32. Lechner CJ, Grüner B, Huang XS, et al. Parasite-specific IL-17-type cytokine responses and soluble IL17 receptor levels in Alveolar Echinococcosis patients. Clin Dev Immunol. 2012; 2012:735342.

33. Wang JH, Goepfert C, Mueller N, et al. Larval Echinococcus multilocularis infection reduces dextran sulphate sodium-induced colitis in mice by attenuating $T$ helper type 1/type 17-mediated immune reactions. Immunology. 2018;154:76-88.

34. Ma XM, Wang L, Zhao H, et al. Th17 cells are associated with the Th1/Th2-cell balance during Echinococcus multilocularis infection. Mol Med Rep. 2014;10:236-40. 
35. Pang NN, Zhang FB, Ma XM, et al. TGF- $\beta /$ Smad signaling pathway regulates Th17/Treg balance during Echinococcus multilocularis infection. Int Immunopharmacol. 2014;20:248-57.

36. Nono JK, Lutz MB, Brehm K. Expansion of Host Regulatory T Cells by Secreted Products of the Tapeworm Echinococcus multilocularis. Front Immunol. 2020;11:798.

37. Wang JH, Müller S, Lin RY, et al. Depletion of FoxP3 ${ }^{+}$Tregs improves control of larval Echinococcus multilocularis infection by promoting co-stimulation and Th1/17 immunity. Immun Inflamm Dis. 2017;5:435-47.

38. Wang JH, Cardoso R, Marreros N, et al. Foxp3 ${ }^{+} \mathrm{T}$ Regulatory Cells as a Potential Target for Immunotherapy against Primary Infection with Echinococcus multilocularis Eggs. Infect Immun. 2018;86:e00542-18.

39. Gottstein B, Wittwer M, Schild M, et al. Hepatic gene expression profile in mice perorally infected with Echinococcus multilocularis eggs. PLoS One. 2010;5:e9779.

40. Lin RY, Lü GD, Wang JH, et al. Time course of gene expression profiling in the liver of experimental mice infected with Echinococcus multilocularis. PLoS One. 2011;6:e14557.

41. Tuxun T, Ma HZ, Apaer S, et al. Expression of Toll-Like Receptors 2 and 4 and Related Cytokines in Patients with Hepatic Cystic and Alveolar Echinococcosis. Mediators Inflamm. 2015:632760.

42. Apaer $\mathrm{S}$, Tuxun $\mathrm{T}, \mathrm{Ma} \mathrm{HZ}$, et al. Expression of toll-like receptor 2, 4 and related cytokines in intraperitoneally inoculated Balb/C mice with Echinococcus multilocularis. Int J Clin Exp Pathol. 2017;10:7947-55.

43. Cocucci E, Meldolesi J. Ectosomes and exosomes: shedding the confusion between extracellular vesicles. Trends Cell Biol. 2015;25:364-72.

44. Tkach $\mathrm{M}$, Théry C. Communication by Extracellular Vesicles: Where We Are and Where We Need to Go. Cell. 2016;164:1226-32.

45. Zheng YD, Guo XL, Su M, et al. Regulatory effects of Echinococcus multilocularis extracellular vesicles on RAW264.7 macrophages. Vet Parasitol. 2017;235:29-36.

46. Buck AH, Coakley G, Simbari F, et al. Exosomes secreted by nematode parasites transfer small RNAs to mammalian cells and modulate innate immunity. Nat Commun. 2014;5:5488.

47. Gagliardi D, Bresolin N, Comi GP, et al. Extracellular vesicles and amyotrophic lateral sclerosis: from misfolded protein vehicles to promising clinical biomarkers. Cell Mol Life Sci. 2021;78:561-72.

\section{Tables}

Due to technical limitations, table 1 is only available as a download in the Supplemental Files section.

\section{Figures}


A

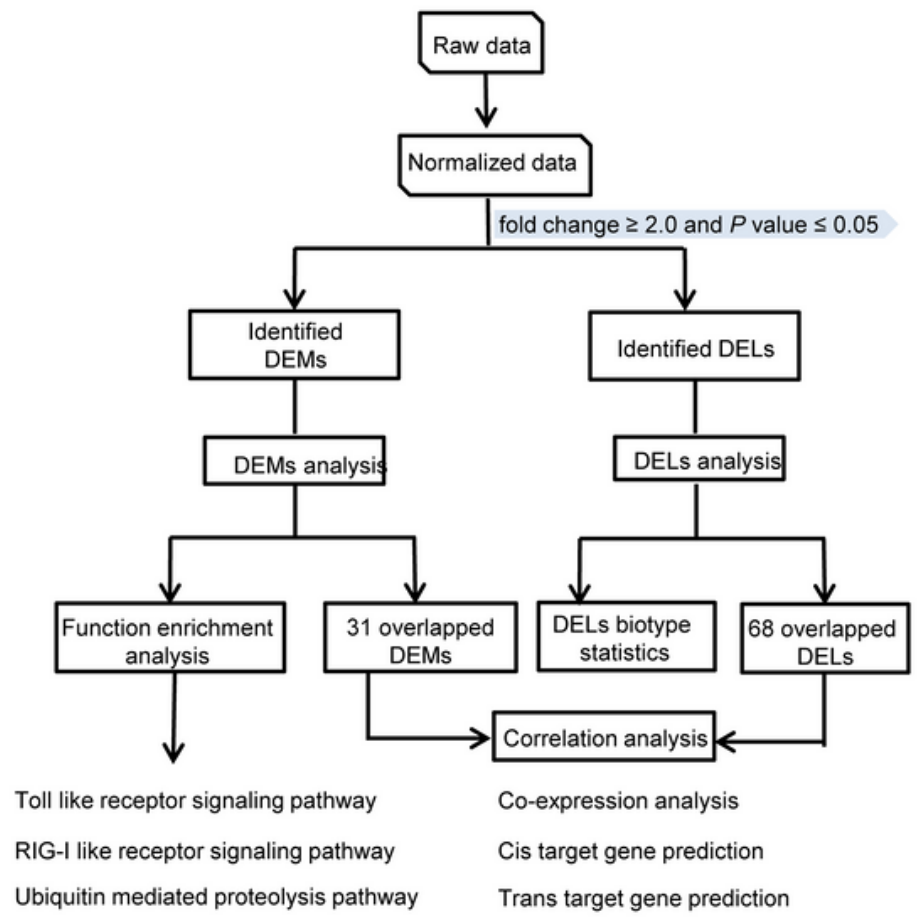

B

a)

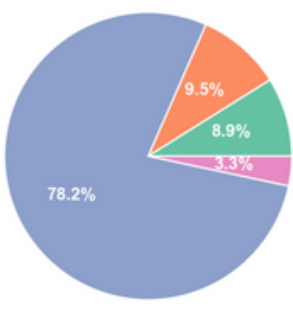

d)

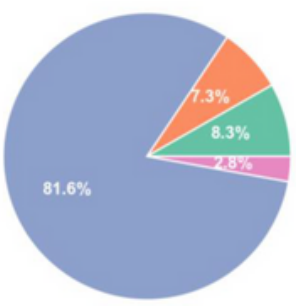

g)

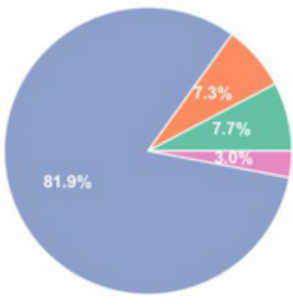

b)

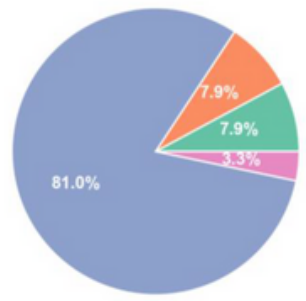

e)

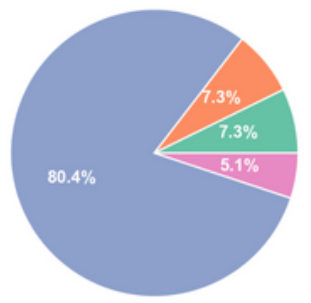

h)

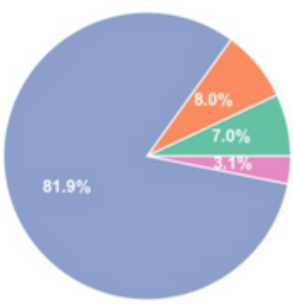

c)

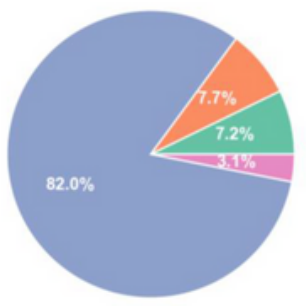

f)

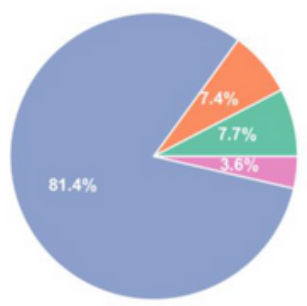

Class_code

- Sense No Exonic

- Antisense

Linc

Exonic

\section{Figure 1}

Overview of RNA microarray analysis a. The workflow for the transcript analysis b. Pie chart of the

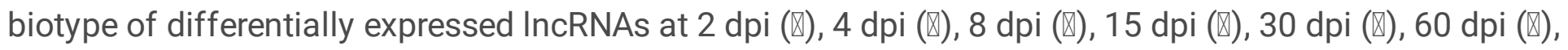

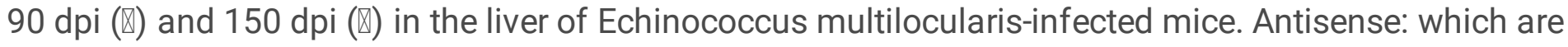
transcribed from the antisense strand, Linc: which locate between annotated protein-coding genes, 
Exonic: which are transcribed from the extron of annotated coding genes and only contain one extron, Sense No Exonic: which not belong to the above three classifications

A

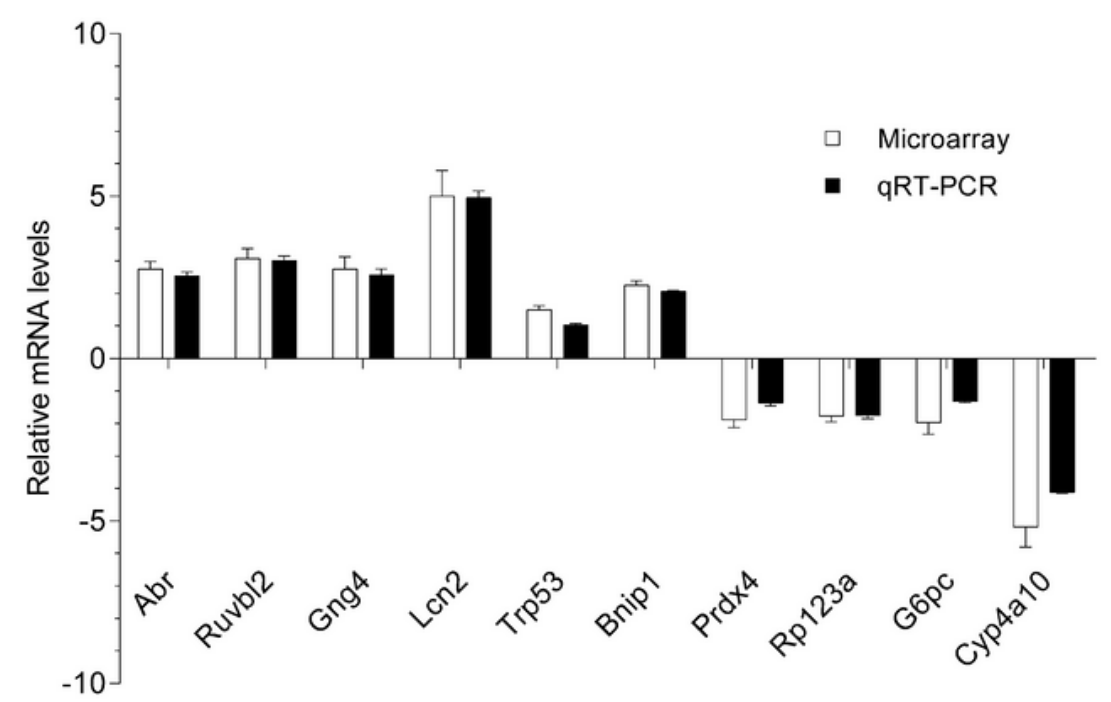

B

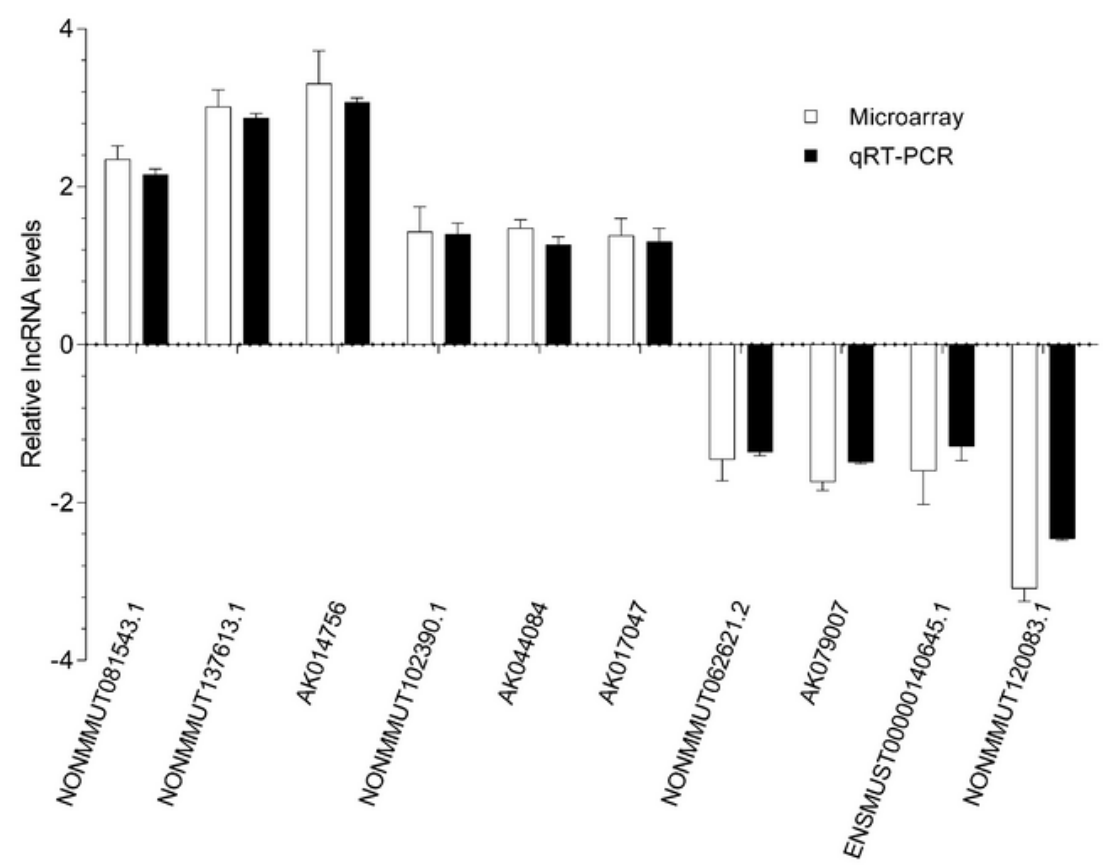

Figure 2

qRT-PCR validation of 20 randomly selected transcripts a. qRT-PCR results of 10 randomly selected differently expressed mRNA $b$. qRT-PCR results of 10 randomly selected differently expressed IncRNA. 
Bars present as mean \pm standard error of three replicates per test in a single experiment repeated three times

A

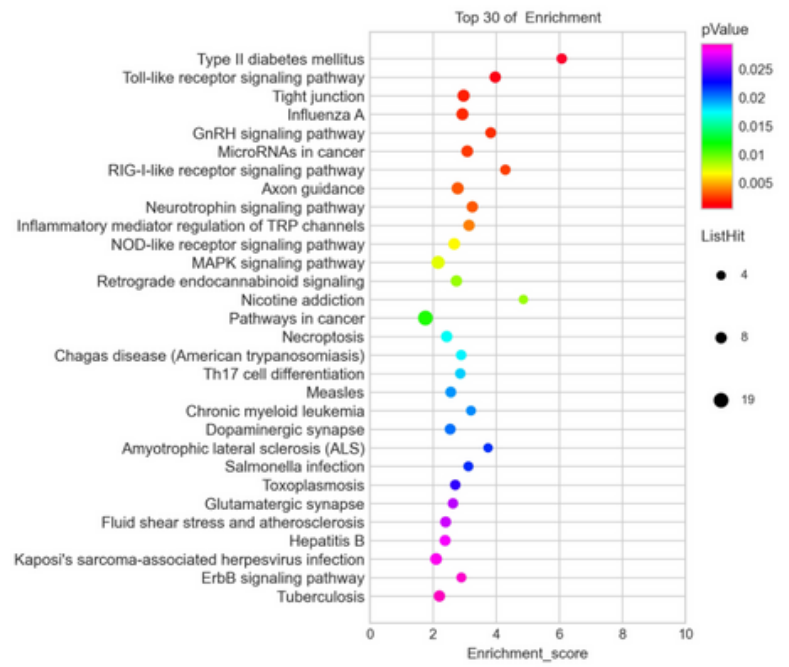

B

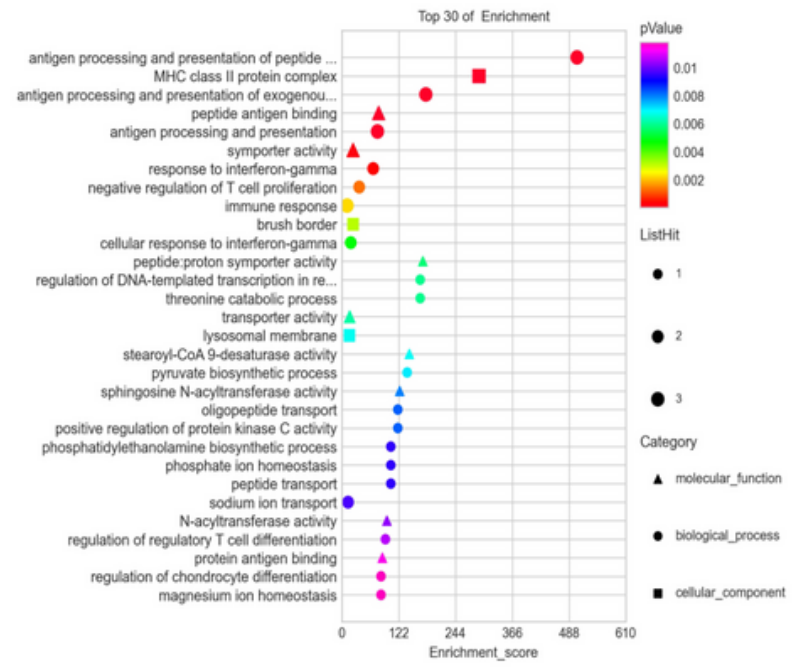

C

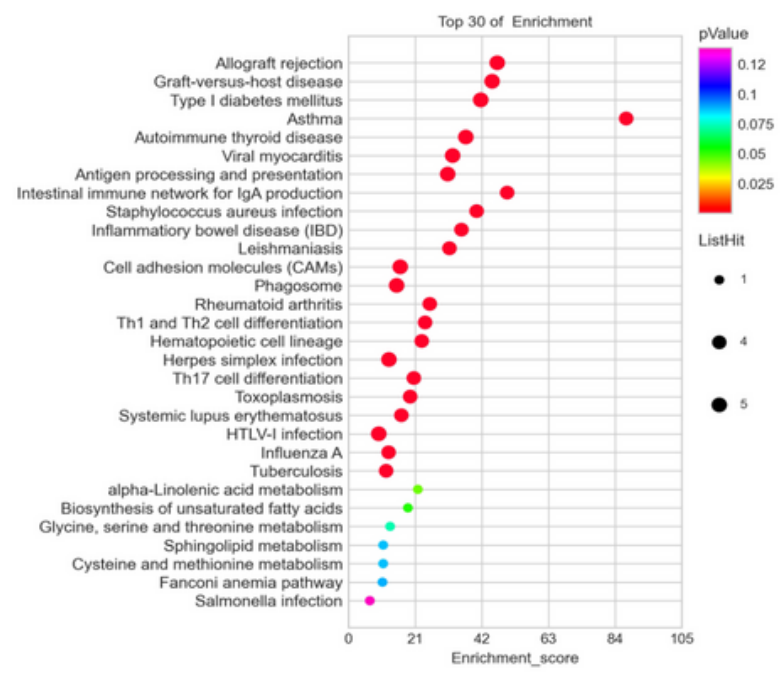

\section{Figure 3}

Functional annotation of the differentially expressed mRNAs and IncRNAs in Echinococcus multilocularis-infected mouse liver a. KEGG analysis of upregulated DEMs at 30 dpi with top 30 enrichment scores b. GO annotation of shared dysregulated mRNAs at eight time points with top 30 
enrichment scores including the domains of biological processes, cellular components, and molecular functions $\mathrm{c}$. KEGG analysis of continuously dysregulated mRNAs at the all eight time points with top 30 enrichment scores

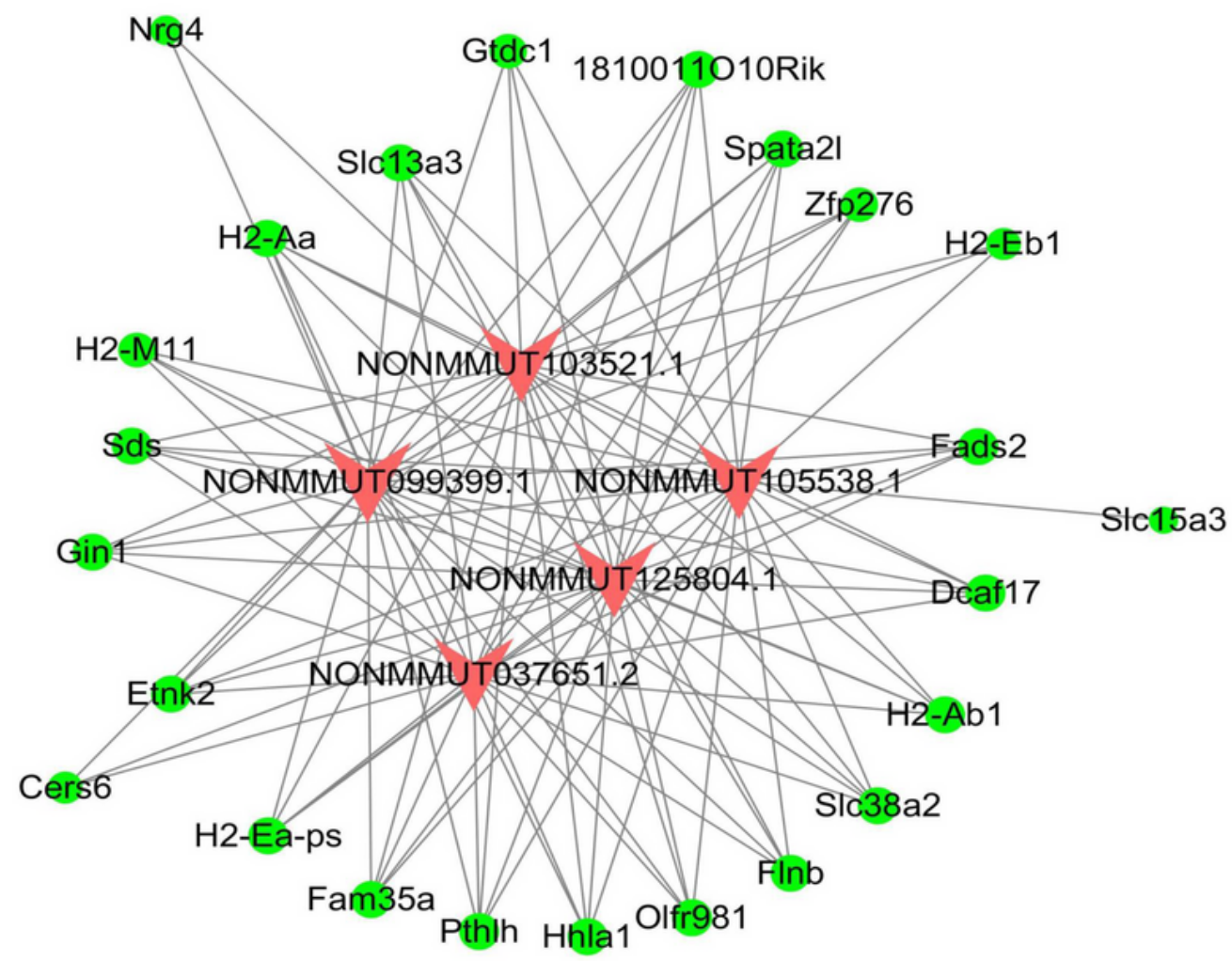

Figure 4

Co-expression network of the shared differentially expressed IncRNAs (DELs) and differentially expressed mRNAs (DEMs) at eight time points in the liver of Echinococcus multilocularis-infected mice. The network 
was based on the Pearson correlation coefficient ( $\mid P C C$ valuel $>0.7, p<0.05)$. The red " $V$ " denotes IncRNAs while the green dot denotes mRNAs.

A

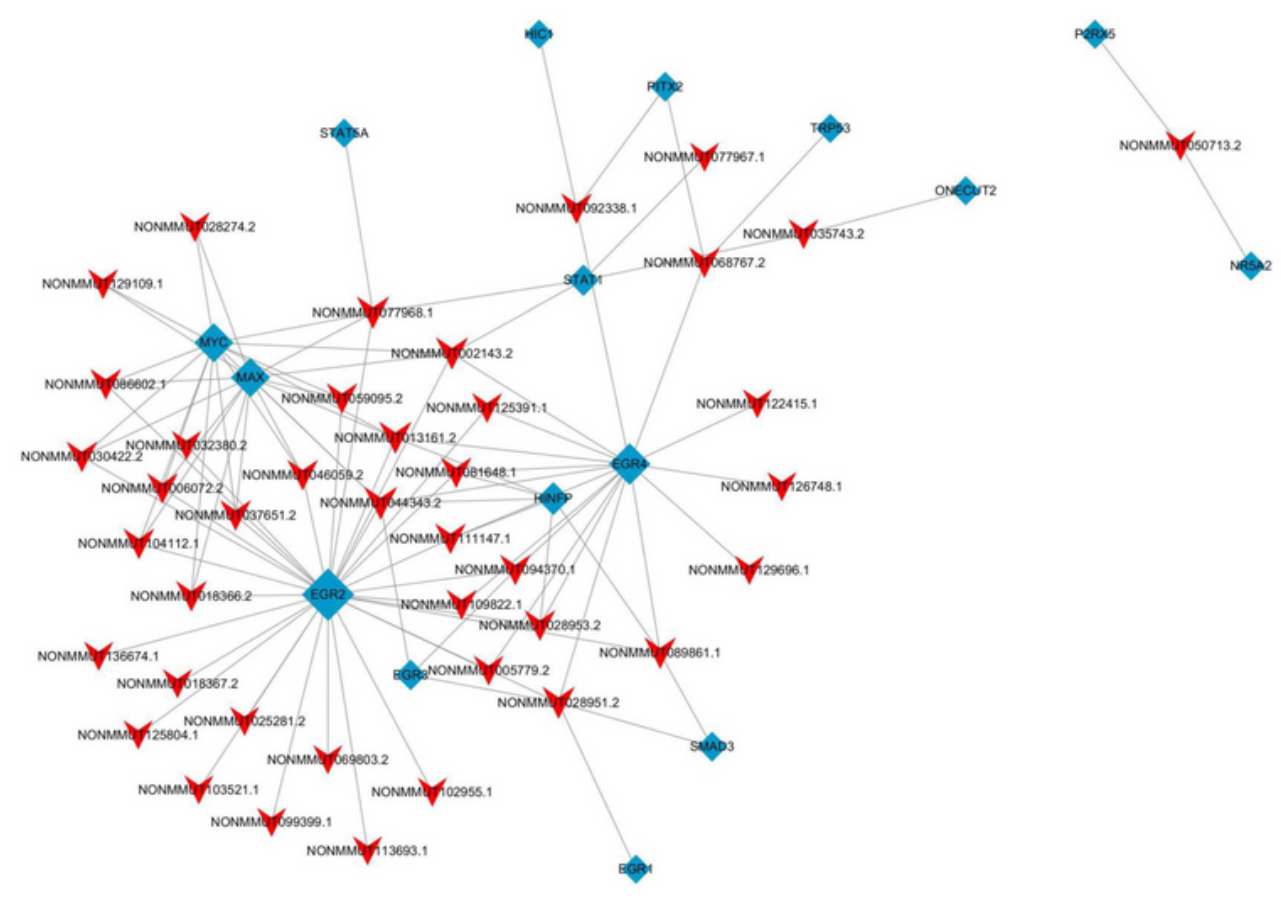

B

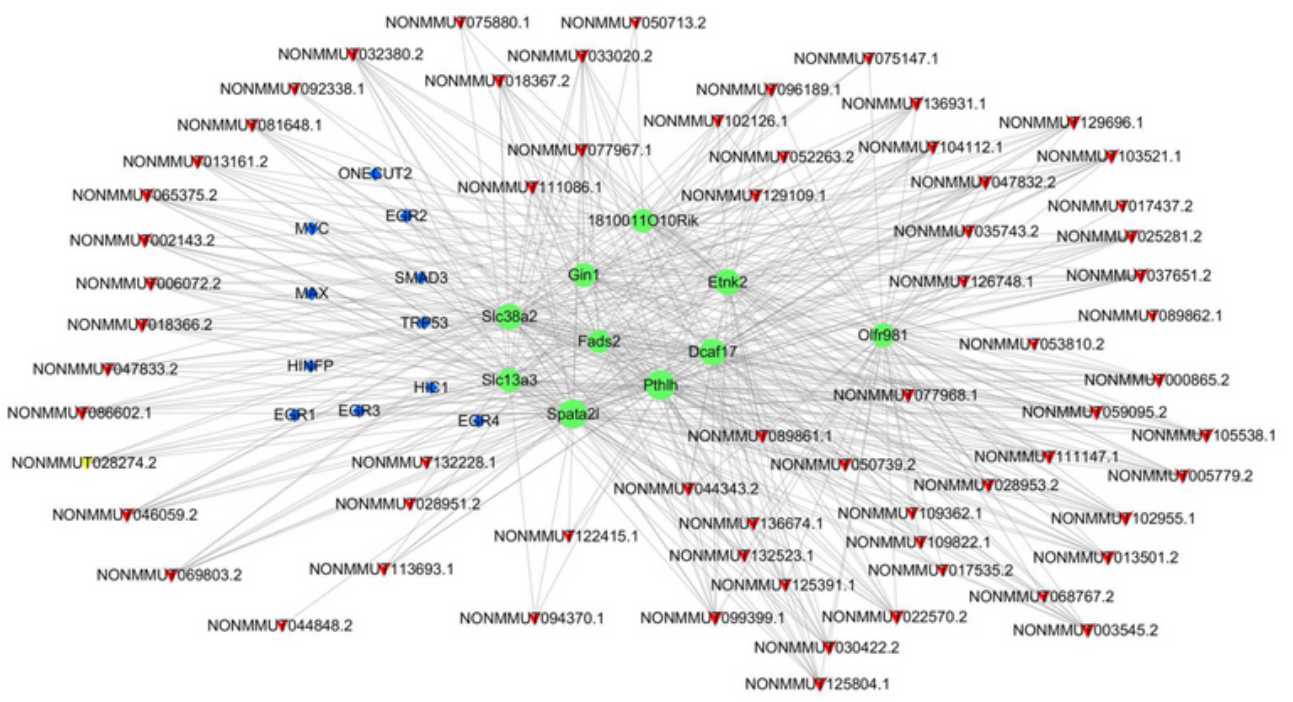

Figure 5

Regulating targets prediction of overlapped IncRNAs and construction of IncRNA-TF-mRNA network a. The network of TFs and continuously dysregulated IncRNAs, consisting of 16 TFs and correlated 41 IncRNAs. The red "V" denotes IncRNAs, while the blue node denotes TF. b. The IncRNA-TF-mRNA network, 
consisting of 66 IncRNAs, 11 TFs and 10 correlated mRNA (according to p-values). The red "V", the blue node and the green node denote the IncRNA, the TF and the mRNA, respectively
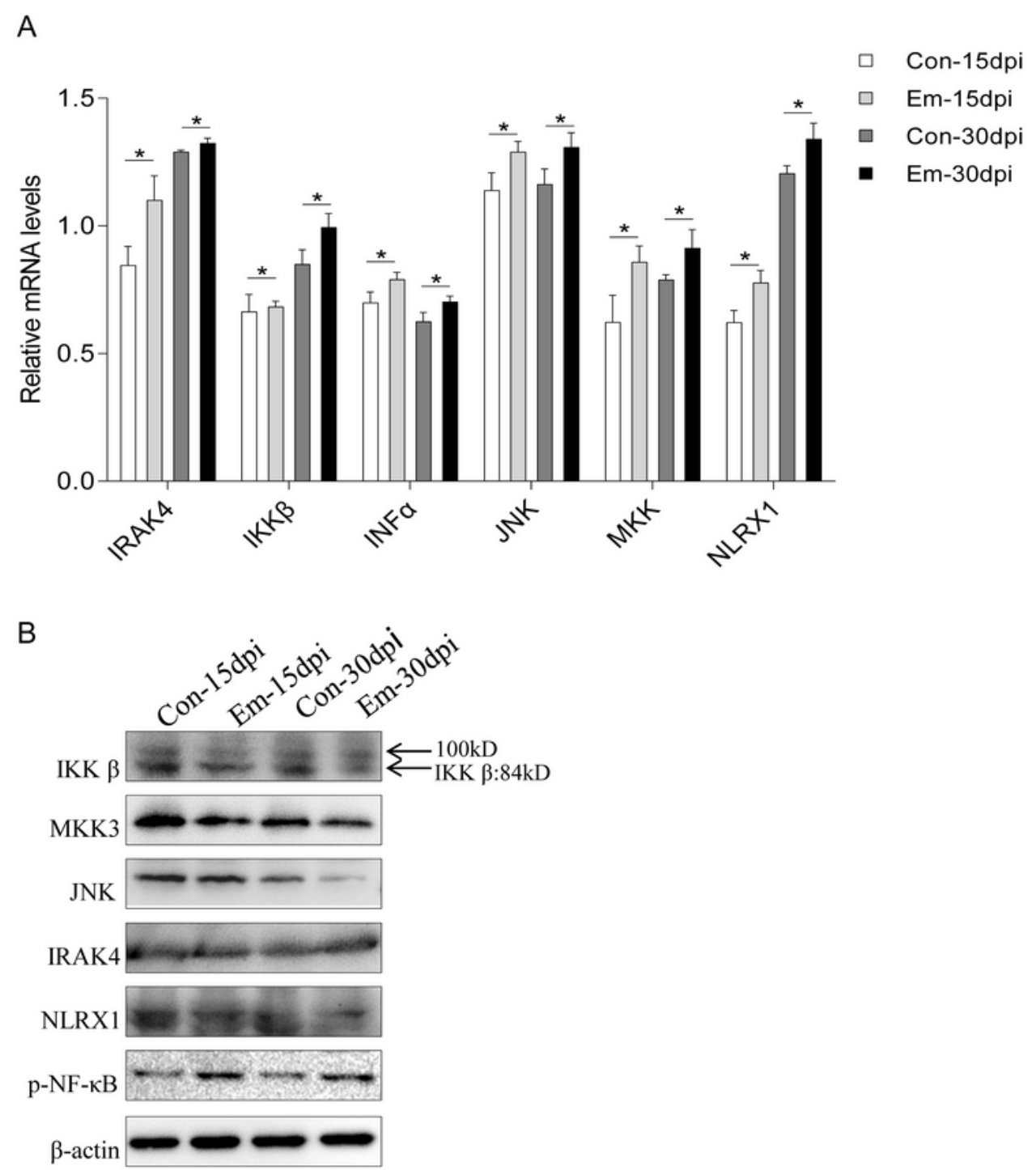

\section{Figure 6}

Validation of six upregulated DEMs involved in TLRs and RLRs signaling pathways a. qRT-PCR results of IRAK4, IKK $\beta$, MKK, JNK, IFN-a, and NLRX1 in Echinococcus multilocularis-infected mouse liver at 15 and $30 \mathrm{dpi}$. The $\mathrm{y}$-axis indicates the value of relative expression level (2- $\Delta \Delta \mathrm{Ct})$ in Standard Deviation of the 
mean expression values. $\beta$-actin was an internal control. * $p<0.05 \mathrm{~b}$. WB results of IRAK4, IKK $\beta$, MKK, JNK, NLRX1, and phospho-NFKB in Echinococcus multilocularis-infected mouse liver at 15 and $30 \mathrm{dpi}$, with $\beta$-actin as a loading control
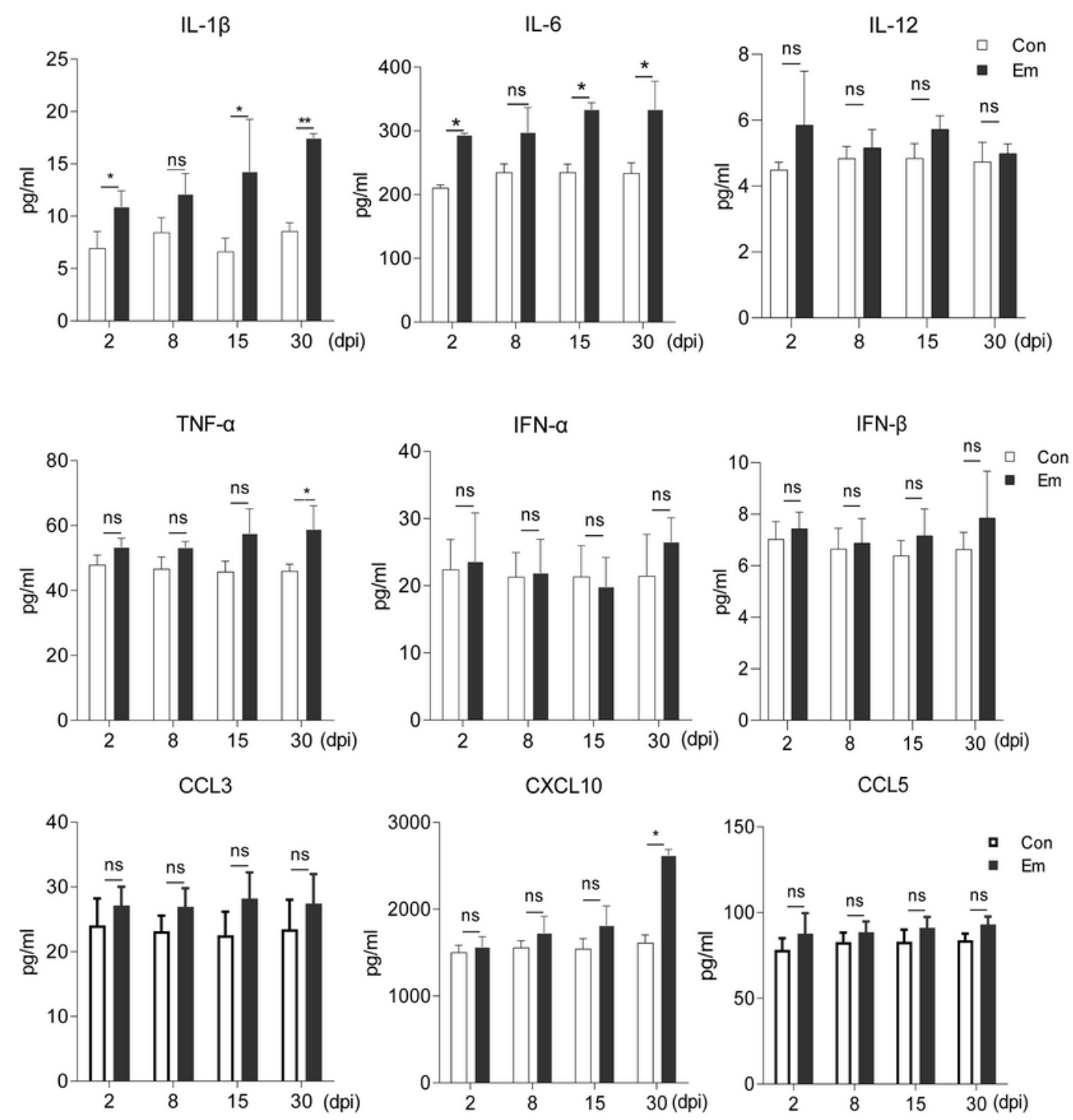

Figure 7

Levels of cytokines and chemokines downstream of the TLRs and RLRs signaling pathway in the serum of Echinococcus multilocularis-infected mouse. ${ }^{*} p<0.05$ 


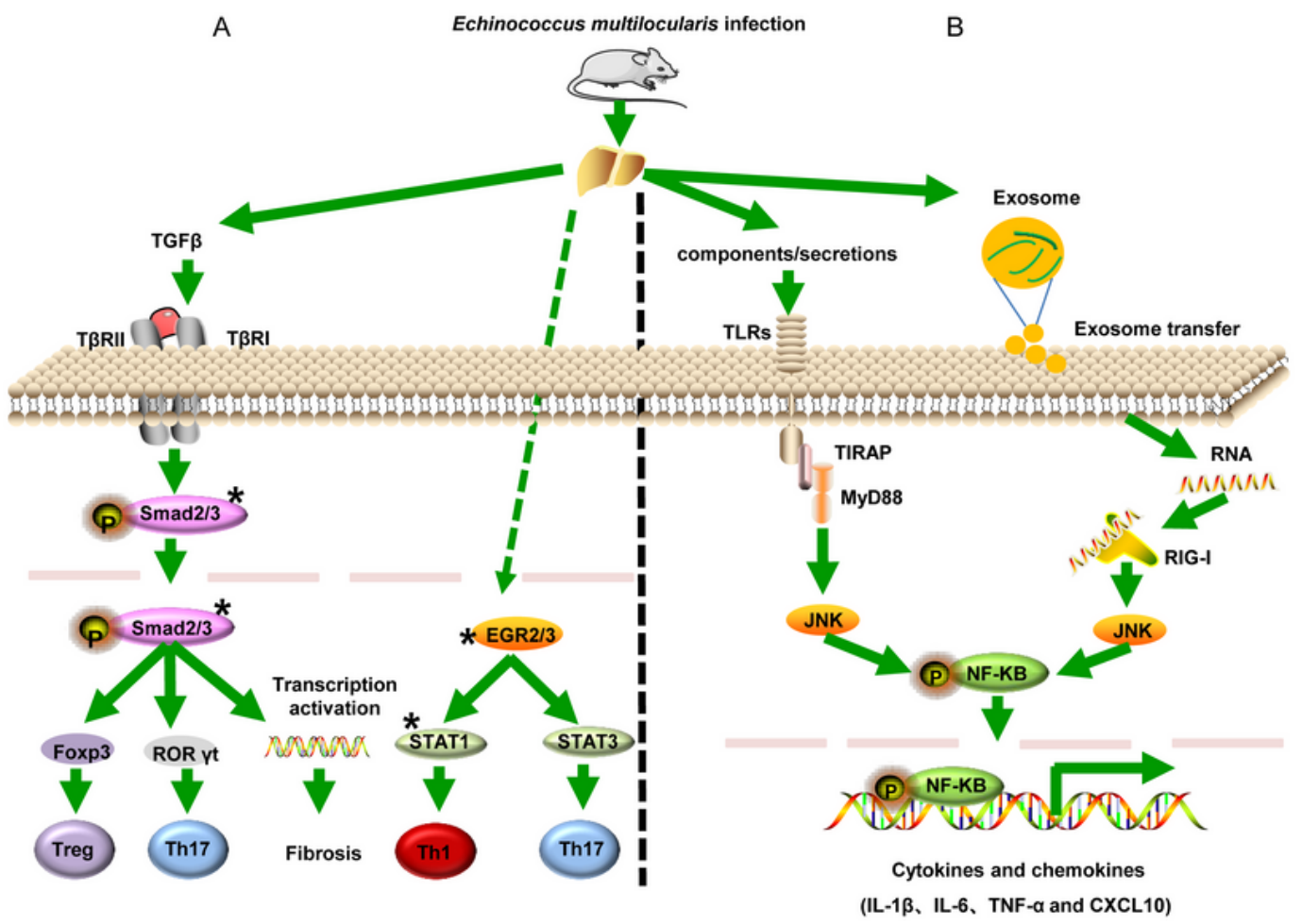

Figure 8

Schematic presentation of predicted targets for differentially expressed IncRNAs in Echinococcus multilocularis-host interplay (a) and the probable influence of Echinococcus multilocularis infection on the TLRs and RLRs signaling pathway of the host (b). * represents the predicted targets

\section{Supplementary Files}


This is a list of supplementary files associated with this preprint. Click to download.

- Table1.tif

- 1AdditionalFigureS1avolcanoplotofDELs.tif

- 10AdditionalfigureS3Microarrayresultsof6genes.tif

- 11Additionaldocumentformethods.docx

- 12AdditionaltableformethodsprimersofDEMandDEL.xIsx

- 2AdditionalFigureS1bvolcanoplotDEMs.tif

- 3AdditionalFigureS1cDELshierarchicalclusteringplot.tif

- 4AdditionalFigureS1dDEMshierarchicalclusteringplot.tif

- 5AdditionalFigureS2aTLRpathwaymmu04620.tif

- 6AdditionalFigureS2bRLRpathwaymmu04622.tif

- 7AdditionalFigureS2cubiquitinat15dpi.png

- 8Additionaltables1aVenn31mRNA.xlsx

- 9AdditionaltableS1bVenn68IncRNA.xIsx 